\title{
Simultaneous Tracking and Registration in SiC/SiC Serial Section Images
}

\author{
Hongkai Yu ${ }^{1}$, Yuxiang Sun ${ }^{1}$, Youjie Zhou ${ }^{1}$, Jeff Simmons ${ }^{2}$, Craig P. Przybyla ${ }^{2}$, and Song Wang ${ }^{1}$ \\ ${ }^{1}$ Dept. of Computer Science and Engineering, University of South Carolina, Columbia, SC, USA \\ ${ }^{2}$ Materials and Manufacturing Directorate, Air Force Research Lab, Dayton, OH, USA
}

Serial section data is acquired by (1) sectioning, (2) surface preparation, and (3) imaging. Often, the imaging apparatus or the sample must be moved periodically, creating registration errors in the asacquired images. For fiber composites, cross-correlation[1] is problematic because many local maxima are produced, often requiring human interaction to manually perform problematic sections. Fiducial marks are often used to mitigate this problem, but require extra work during acquisition. In this work, we develop an unsupervised technique that combines the tracking and registration processes into a single algorithm that eliminates the need for cross-correlation or fiducial marks. The algorithm is iterative, alternating between tracking and registering, optimizing both steps simultaneously, and giving fully tracked fiber architectures.

For this study, a series of serial section images, Figure 1, of a continuous SiC fiber reinforced SiNC matrix ceramic matrix composite (CMC), S200 (www.coiceramics.com/nonoxidepg.html), were obtained by automated serial sectioning with RoboMet.3D ${ }^{\mathrm{TM}}(\mathrm{UES}$, Inc.) with a nominal pixel size of $0.5 \mu$ and an inter-section distance of $1 \mu$. Fiber centers were detected with a modified Hough transform[2] to give fiber centers, section by section. The accuracy of detecting the centers was estimated to be $94 \%$.

The algorithm developed is summarized in Algorithm 1. We assume that there is a 'true' location of each fiber and that the detected position differs from the true position because of (1) noise in the detec-tion algorithm, (2) false negative and false positive detections, and (3) the shift due to misregistration. We have addressed the first two of these in other publications[1, 3], and address the third here.

We model this with a shift vector, $\mathbf{s}$, such that the detected position is the sum of its true position and the shift vector:

$$
\tilde{\mathbf{t}}=\mathbf{t}+\mathbf{s}
$$

where $\tilde{\mathbf{t}}$ is the detected position and $\mathbf{t}$, the true position. $\mathbf{s}$ is a latent variable to be estimated.

The values of $\tilde{\mathbf{t}}$ are, alternatively, corrected by the Kalman filter for consistency and by a linear regression fit for straightness (over distances on the order of the inter-section distance). The difference between $\tilde{\mathbf{t}}$ and the regression-fit centers, $\hat{\mathbf{t}}$, forms an 'energy penalty,' $E$, to be minimized:

$$
E=\frac{1}{N K} \sum_{j=1}^{N} \sum_{i=1}^{K}\left\|\hat{\mathbf{t}}_{i}^{(j)}-\tilde{\mathbf{t}}_{i}^{(j)}\right\|^{2}
$$

where $N$ is the number of sections, $K$, the number of fibers that pass the 'sanity check' of being detected in every layer, subscripts refer to fiber locations, and superscripts to sections. E is a convex function in $\mathbf{s}$, so it may be minimized by gradient descent to give the estimate of the shift vector, $\hat{\mathbf{s}}$, which is used to update the values of $\tilde{\mathbf{t}}$. These are fed back to the Kalman filter, and the process repeated until convergence. The tracking accuracy (MOTA)[4] and energy $(E)$ are shown in Figure 2. 
References:

[1] Youjie Zhou, et al. Large-scale fiber tracking through sparsely sampled image sequences of composite materials. IEEE Transactions on Image Processing, 25(10):4931-4942, 2016.

[2] C Przybyla, et al. Automated image processing techniques and fiber identification for three dimensional characterization of fiber reinforced composites. In ASC 29th Technical Conference, 16th US-Japan Conference on Composite Materials, \& ASTM D30 50th Anniversary meeting, 2014.

[3] Hongkai Yu, et al. Groupwise tracking of crowded similar-appearance targets from low-continuity image sequences. In Proceedings of the IEEE Conference on Computer Vision and Pattern Recognition, pages 952-960, 2016.

[4] Keni Bernardin and Rainer Stiefelhagen. Evaluating multiple object tracking performance: the clear mot metrics. Journal on Image and Video Processing, 2008:1, 2008.

[5] James Munkres. Algorithms for the assignment and transportation problems. Journal of the society for industrial and applied mathematics, 5(1):32-38, 1957.

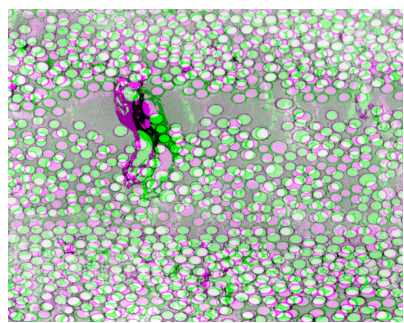

(a)

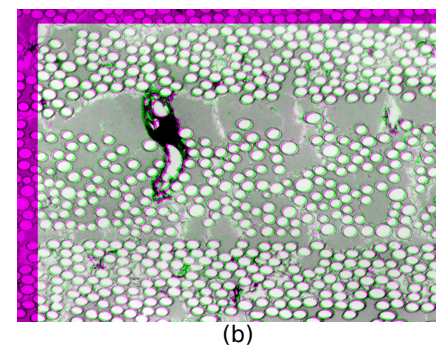

(b)

Figure 1. Two registered layers, bottom image in color. (a) As acquired. (b) registered with our algorithm.

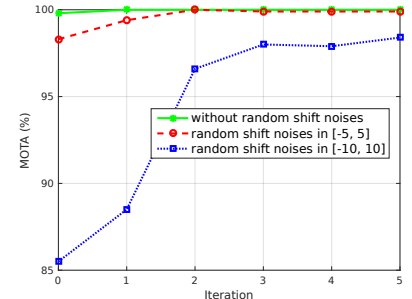

(a)

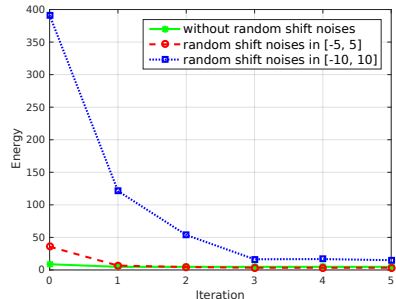

(b)

Figure 2. Algorithm performance. (a) MOTA tracking accuracy, higher values are better, (b) energy penalty $E$.

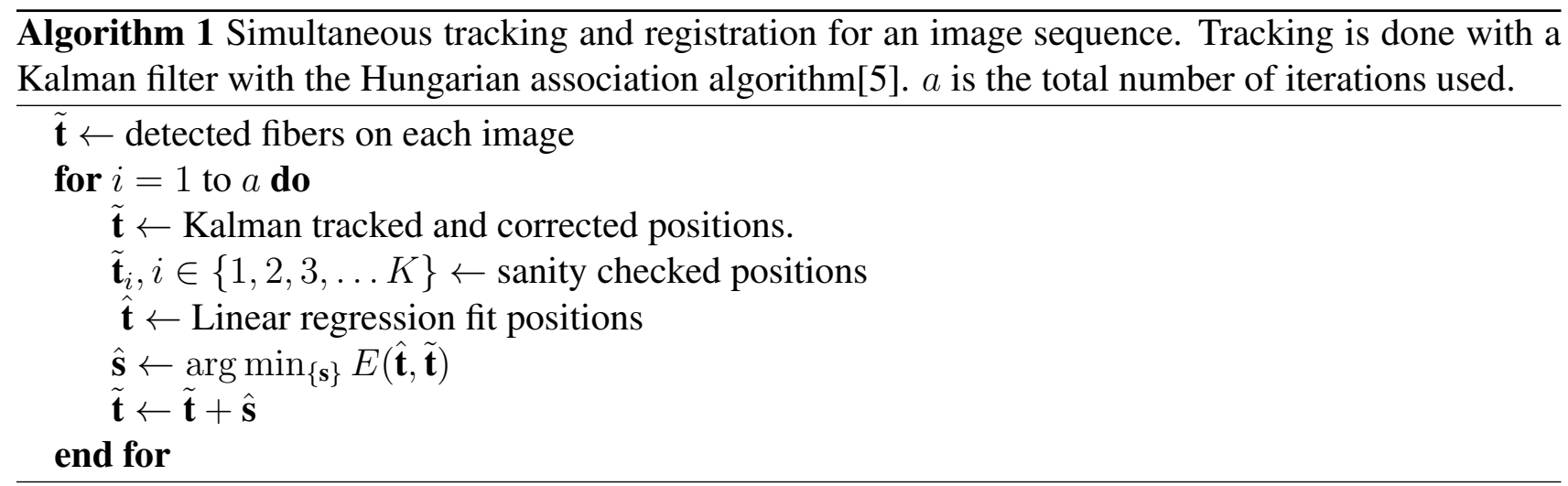

